Crise(s) dans le monde ibérique et ibéro-américain

\title{
Nadie ama a un policía de Guillermo Orsi et La última caravana de Raúl Argemí
}

Regards sur la crise argentine de 2001

\section{Maud Gaultier}

\section{OpenEdition}

\section{Journals}

Édition électronique

URL : https://journals.openedition.org/cher/3630

DOI : $10.4000 /$ cher.3630

ISSN : 2803-5992

\section{Éditeur}

Presses universitaires de Strasbourg

Édition imprimée

Date de publication : 1 décembre 2015

Pagination : 105-116

ISBN : 978-2-86820-913-9

ISSN : 1968-035X

\section{Référence électronique}

Maud Gaultier, « Nadie ama a un policía de Guillermo Orsi et La última caravana de Raúl Argemí », reCHERches [En ligne], 15 | 2015, mis en ligne le 01 décembre 2021, consulté le 22 décembre 2021. URL : http://journals.openedition.org/cher/3630 ; DOI : https://doi.org/10.4000/cher.3630

\section{(c) (i) (3) (2)}

Ce(tte) œuvre est mise à disposition selon les termes de la Licence Creative Commons Attribution -

Pas d'Utilisation Commerciale - Partage dans les Mêmes Conditions 4.0 International. 


\title{
Nadie ama a un policía de Guillermo Orsi et La última caravana de Raúl Argemí
}

\author{
Regards sur la crise argentine de 2001
}

\author{
MAUD GAULTIER \\ Aix Marseille Université (CAER)
}

\begin{abstract}
T 'association entre société en crise et roman noir semble bien souvent aller de soi : nombreuses sont les études retraçant l'histoire du genre qui présentent la crise mondiale de 1929 comme lévénement ayant engendré la naissance du policier «dur », avec la publication par Dashiell Hammett du roman, qui apparut comme le premier «hard-boiled», Moisson rouge. D’autres critiques ont montré que l'on peut situer l'émergence du genre quelques années auparavant, et parlent alors d'une "erreur chronologique». Cependant, ils soulignent aussi qu'il n'est pas innocent que cette "erreur» ait perduré: celle-ci instaure la crise comme une dimension quasiment constitutive du policier. Le Magazine littéraire de mai 2012, qui dresse un bilan du "polar aujourd'hui», présente le genre comme le «chroniqueur de la crise». Il apparaît non seulement, dès son origine, comme un «chroniqueur du réel», mais aussi comme à la fois un produit de la crise, et un chroniqueur de cette même crise dont il serait issu.

Dans un article récent, le journal argentin Clarín faisait état de l'actuel «boom» du policier national, soulignant «le nouvel essor indiscutable du genre noir et policier en Argentine». Il explique ce boom justement par la crise vécue par le pays en $2001^{1}$ : non seulement une génération nouvelle de jeunes écrivains

1 «El nuevo e indiscutible auge del género negro y policial en Argentina tiene su explicación. El crimen sigue siendo un eslabón indiscutido de la sociedad y el avance de las desigualdades sociales -con un crecimiento exponencial en la década del 90- ha posibilitado la aparición de un conjunto de narradores que supieron amalgamar la realidad con la literatura para crear obras que son un espejo de la época.» http://www. clarin.com/sociedad/Boom-policial-autores-festivales-internacionales_0_678532260. html (consultée le 09/12/2013).
\end{abstract}


se serait presque «naturellement» tournée vers un genre apte à refléter les temps actuels, mais le lectorat, ainsi que les maisons d'édition, semblent accueillir de plus en plus favorablement les nouveaux polars. Tous ne deviennent pas des best sellers, comme ce fut le cas pour Las viudas de los jueves de Claudia Piñeiro (2005), dont nous parlerons un peu ici, mais certains auteurs, qui parfois même n’avaient jamais été publiés jusqu'alors, se sont vus propulsés sur le devant de la scène éditoriale. Aussi, au sein de cette littérature que nous pouvons appeler de "post-crise», le genre noir est-il particulièrement présent.

Le corpus de romans policiers argentins parus après la crise et faisant de celle-ci, sous une forme ou sous une autre, le centre des récits, est donc particulièrement vaste. Vaste quantitativement, d'une part, mais aussi par sa diversité: en effet, s'il est bien question dans tous les cas de romans proposant une intrigue policière en rapport étroit avec la dernière crise argentine, l'écriture réaliste d'un Leonardo Oyola, dans Gólgota (2008), diffère radicalement du pari fictionnel, tendant vers le fantastique, d'un César Aira dans Las noches de Flores (2004), ou encore, pour ne citer que ces trois exemples parmi un ensemble extrêmement foisonnant, de la peinture de la crise par Néstor Ponce qui, dans son roman Azote, allie roman noir et science-fiction (2008). Il n'y a donc pas une écriture policière de la crise, mais bien des écritures de la crise, dont nous avons choisi d'étudier une des modalités à travers l'étude de deux romans: Nadie ama a un policía de Guillermo Orsi, paru en 2007, et La última caravana de Raúl Argemí, paru en $2008^{2}$.

Raúl Argemí et Guillermo Orsi possèdent plusieurs points en commun: tous deux nés en 1946, ont été réellement découverts dans les années 2000, en Espagne, grâce à l'obtention de plusieurs prix littéraires. Traduites dans plusieurs langues, leurs œuvres sont paradoxalement davantage diffusées en Europe qu'en Amérique Latine. Si l'un d'eux, Raúl Argemí, vit désormais en Espagne (Guillermo Orsi vit dans la province argentine de Córdoba), le regard qu'ils portent dans leurs romans sur la crise de 2001 se fait clairement depuis leur identité argentine. C’est ce double regard, posé depuis la fiction policière, que nous allons mettre en lumière.

\section{Trame et crise}

Nadie ama a un policía débute de manière assez classique par l'irruption d'un meurtre dans l'entourage du personnage principal, un ancien policier reconverti, après la dernière dictature, en vendeur de sanitaires. Le moteur de l'action sera donc les efforts du personnage pour retrouver les assassins, et comprendre les

2 Nous avons essentiellement choisi de focaliser notre attention sur ces deux œuvres, mais nous mènerons cette étude sans nous priver d'en citer d'autres, ce qui nous permettra d'appuyer ou de mettre en perspective nos analyses de la crise vue par les deux romans. Nous ferons donc appel en particulier à Ernesto Mallo, Néstor Ponce et Claudia Piñeiro. 
raisons du meurtre. Pendant cette enquête, il va être rattrapé à la fois par un passé qu'il avait décidé de fuir, et par la crise qui secoue le pays, puisque le mobile du meurtre sera en rapport avec cette crise, tout comme d'ailleurs le «mobile» du roman lui-même, c'est-à-dire son origine, comme nous invite à le penser l'appareil paratextuel.

Le point de départ de La última caravana est une fille qui retrouve son père ayant volontairement disparu. Le roman est constitué par le récit de ce père racontant la vie qu'il a menée pendant cette absence. Si chez Guillermo Orsi, la narration est entièrement prise en charge par le personnage principal, en focalisation interne, Raúl Argemí fait ici intervenir deux narrateurs: la troisième personne, largement minoritaire, alterne avec le récit à la première personne du personnage principal qui, à l'instar de celui de Nadie ama a un policía, se trouve lui aussi obligé de revenir sur un passé qu'il avait décidé d'oublier. Il raconte ainsi à sa fille principalement son été 2001, pendant lequel, pour financer la création d'un nouveau parti politique, il a fait un «casse» dans une banque. Une banque dans laquelle il n'y avait plus d'argent, crise oblige...

Ces trames prennent tout leur sens grâce à l'immersion du personnage dans la crise. Même si les fréquentes prolepses et analepses confèrent aux romans un cadre temporel beaucoup plus ample, les histoires se déroulent très précisément pendant la crise, c'est-à-dire pendant l'été 2001. Mais la crise n'est pas seulement une toile de fond contextuelle. L'intrigue, qui met en scène des personnages fictionnels, semble presque s'effacer au profit du récit d'une autre histoire: celle de la crise, sous tous ses aspects. À la fois financière et économique, politique et institutionnelle, et bien sûr sociale, cette crise apparaît d'une ampleur et d'une profondeur sans précédent, dans une Argentine pourtant déjà secouée auparavant par de nombreuses crises. Les romans reviennent sur les faits euxmêmes, qui sont amplement décrits dans les deux œuvres, en décryptent les mécanismes, les raisons, les conséquences et les prolongements.

Ce que nous appelons les faits eux-mêmes, ce sont les divers événements du mois de décembre 2001 qui amènent le président Fernando de la Rúa à décréter l'état de siège, déclenchant ainsi une révolte populaire et spontanée qui le forcera à démissionner et à quitter en hélicoptère la "casa rosada", siège du pouvoir exécutif. Les romans retracent ainsi, par bribes, comme autant d'épisodes insérés dans la narration, les moments clé de la crise: "saqueos", "corralito", "cacerolazo $"^{3}$, la violente répression policière au lourd bilan (une quarantaine de morts), la succession des cinq présidents en moins de deux semaines, etc.

Pourtant, aucun des protagonistes n'est réellement partie prenante des événements. Ils appartiennent à la classe moyenne, voire presque pauvre dans le cas d'Argemí dont le personnage est un fonctionnaire qui n'a pas été payé depuis des mois. Ils sont comme tout le monde, victimes des mesures prises

3 Pour une explication de ces termes, voir Diccionario de la crisis (Gobello J./Oliveri M.H. 2002). La bibliographie sur la crise elle-même est extrêmement abondante. Nous citons quelques références en fin d'article. 
par le gouvernement. Cependant, n'ayant parfois accès aux événements que par la radio ou les journaux, ils demeurent avant tout des observateurs du désespoir de la population impuissante: «Los noticiosos daban cuenta de la histeria de la gente, a la que le habían limitado el retiro de dinero en efectivo de los bancos. El país se preparaba para caer una vez más en picada, y a los giles les anunciaban que se ajustaran los cinturones y ni chistaran» (Orsi 2007 : 142). Ou encore: «La gente, los diarios, vaya uno a saber quién, llamó "el corralito", a ese acorralamiento de su dinero. Y no se podía hacer otra cosa que insultar a los cajeros automáticos, que en pocas horas sólo daban respuestas técnicas, justificando sus panzas vacías» (Argemí 2008: 254).

Le «cacerolazo » est décrit comme un bruit lointain parvenant au protagoniste de Nadie ama a un policía à travers les fenêtres ouvertes de son appartement:

Por las ventanas abiertas llegaba el ruido lejano de la percusión sobre cacerolas, columnas de alumbrado y todo lo que resonara, gritos y cánticos, vecinos que marchaban desvelados por el enojo y el calor, confluyendo en la plaza de mayo como siempre que algo resquebraja en la Argentina (Orsi 2007: 223).

De manière analogue, dans La última caravana, il est bien indiqué que le personnage ne se trouvait pas au milieu de la foule, au moment où «la clase media sacaba cacerolas a la calle para protestar con ruido porque los bancos no soltaban sus ahorros» (Argemí 2008:254).

Les personnages de ces deux romans décrivent les scènes de débordement populaire avec un regard souvent désabusé, et même avec un humour et une distance frisant l'ironie, qu'ils n'auraient sans doute pas eus s'ils avaient été partie prenante des événements. Cela n'empêche évidemment pas les récits de souligner le caractère dramatique de la situation. Raúl Argemí et Guillermo Orsi utilisent d'ailleurs tous deux la métaphore de l'Apocalypse: «Los peatones que vienen atrás nos atropellan y nos apartan de su paso sin pedir disculpas, ensimismados en las imágenes que perciben del inminente apocalipsis...» (Orsi 2007: 212). Ou bien: «El país que se hunde, y la bolsa que sube; ¿Qué necesita para ver que se viene el Apocalipsis?»(Argemí 2008: 280)4.

La última caravana et Nadie ama a un policía apparaissent comme les chroniqueurs acides d'une apocalypse plongeant l'Argentine dans un état de sidération absolue. Histoire collective et histoire individuelle s'entremêlent dans un savant tissage narratif qui permet d'incorporer au récit de très nombreuses descriptions de la crise et de non moins nombreux commentaires sur la crise, sans pour autant leur donner l'allure de digressions. C'est bien là que réside la force des récits, dans la mesure où c'est ce qui donne au genre noir sa véritable raison d'être et toute sa dimension. En effet, au-delà de l'anecdote fictionnelle racontée,

4 Notons que cette apocalypse fait justement l'objet du roman d'anticipation de Néstor Ponce, Azote, qui situe les événements en 2018, lors d'une crise appelée la "gran depresión", et nous plonge dans un univers où la classe moyenne a disparu. $\mathrm{Ne}$ restent que quelques îlots de personnes privilégiées, vivant dans des zones fermées, dans une pauvreté extrême. 
les romans semblent entreprendre en réalité le récit d'un seul véritable crime: la crise elle-même, dont la victime n'est autre que le peuple argentin. Dans cette perspective, le genre noir ne serait pas seulement un genre destiné à entretenir avec la réalité sociale, et en particulier avec les aspects sombres de nos sociétés, des liens étroits; nous pouvons dire que les romans noirs instaurent quasiment en système d'écriture la question même de la criminalité. Nous comprenons dès lors pourquoi ils connaissent un tel essor à l'aube de ce troisième millénaire, qui a débuté si tragiquement en Argentine par la crise de 2001.

\section{Un retour sur les années quatre-vingt-dix}

Si finalement, le seul véritable crime, au sein des romans, n'est autre que la crise elle-même, la question est alors de savoir qui est, ou plutôt, qui sont les coupables. Pour le savoir, ne suffit-il pas de se demander à qui profite la crise? L'une des réflexions du narrateur de Nadie ama a un policía semble apporter des éléments de réponse: "Y hay asesinos, claro. Gente entrenada para matar por encargo, tropas de élite que ya no se pintan la cara ni se enmascaran con pasamontañas, sino que se afeitan y se bañan en aguas de colonia que compran en París. De ellos no se habla...» (Orsi 2007: 156). C'est bien la vocation de ces deux romans: mettre en scène les "vrais coupables", ceux dont "personne ne parle».

Il va falloir d'abord les chercher dans le passé récent, c'est-à-dire les «années Menem», présentées à l'époque par la plupart des médias comme celles du «miracle économique». Guillermo Orsi les décrit en ces termes:

El mundo opulento aplaudía mientras tanto el milagro económico y político del gobierno más corrupto de la tierra, que había llegado al poder prometiendo exactamente lo contrario de lo que haría y financiado por el narcotráfico, sin que a los gendarmes del norte se les moviera un pelo. En menos de una década, ese gobierno ejemplar transformó a la Argentina en una gran tienda de saldos, materiales y humanos. (Orsi 2007: 251)

Comme on pouvait s'y attendre, la corruption, omniprésente dans les deux romans, se retrouve à tous les niveaux de la société. Elle aboutit à la paupérisation d'une frange de la population, symbolisée par la figure du "cartonero». Un certain nombre de pages est consacré à dépeindre cette misère, résultat direct du manque de scrupules des classes dirigeantes: "El estado no gasta demasiado en atender a los pobres. Gasta fortunas, me corrigió el juez, el presupuesto destinado a salud es enorme, pero la plata queda en el camino, en los bolsillos y las cuentas secretas de la mafia que nos gobierna» (Orsi 2007: 242).

Mais surtout, est mise en relief, comme d'ailleurs dans Las viudas de los jueves de Claudia Piñeiro, la fracture grandissante entre une classe moyenne qui profite illusoirement du système économique mis en place, et une population marginalisée qui ne cesse de s'appauvrir: «La bolsa siempre cerraba en alza. El ingreso por cabeza estaba al nivel del Primer mundo. Muchos cartoneros habían 
comenzado a salir de día, porque de noche había mucha competencia.» (Argemí 2008: 211).

Si la politique de Menem est remise en cause dans son ensemble, une mesure en particulier cristallise toutes les autres: la fameuse loi de 1991, «el uno a uno », dite loi de "convertibilité», décrétant l'égalité de l'unité monétaire argentine avec le dollar. Si cette mesure prétendait répondre à l'endettement excessif du pays $^{5}$, les personnages n'y voient quant à eux qu'une stratégie d'enrichissement par la corruption:

[El uno a uno] es un truco de cuarta categoría, Roque, cuando se terminen las reservas se va todo al carajo. ¿Entonces qué? ¿Dónde está el negocio? ¿Dónde puede estar? En la especulación financiera, si tenés mucho dinero, y en las comisiones, si estás en el gobierno! (Argemí 2008: 124-125).

Cette mesure a cependant créé l'illusion d'une prospérité économique. Claudia Piñeiro met en lumière les mécanismes du "uno a uno", en montrant drabord comment la mesure «había reactivado el mercado»(2005: 41). Permettant aux riches de spéculer, elle déboucha d'abord sur: "la euforia del bienestar de los noventa». Au cours de cette période, comme le dit l'un de ses personnages: "la gente que podía invertir estaba contenta» (2005: 82-83). Les classes moyennes se sont alors adonnées à un consumérisme effréné, à l'origine même du drame qui sous-tend le roman de Piñeiro. On retrouve dans $L a$ última caravana et Nadie ama a un policía la même dénonciation du mirage, du mensonge même, qu'a pu représenter une telle mesure, tant pour les classes aisées que pour les classes moyennes:

Las fábricas cerraban, o se convertían en vendedoras de chicles de Bora Bora, pero sin embargo la gente estaba contenta. Eso demostraba, decían, que el peso era una moneda fuerte gracias al "uno a uno", y se iban de vacaciones a Miami

affirme le narrateur du roman d'Argemí (2008: 212). Le protagoniste de Nadie ama a un policía met tout autant en exergue cette illusion de l'argent facile:

La ciudad estaba espléndida, relucía bajo el sol, las calles atestadas, los comercios rebosantes, mucha gente comprando televisores, heladeras y hasta autos, como quien compra galletitas en el kiosco de la esquina. Los que tenían efectivo compraban dólares o artículos de alto costo (Orsi 2007: 147).

Ainsi, si la corruption règne au plus haut niveau de l'Etat, Raúl Argemí et Guillermo Orsi ne se bornent pas à pointer du doigt les classes dirigeantes et aisées, ils dépeignent toute une société, certes en grande partie victime, mais aussi quelque peu complice du piège dans laquelle elle est tombée. En cela, ils rejoignent Claudia Piñeiro, malgré la différence de leurs écritures.

5 Si tous les économistes s'accordent pour dire que la mesure a eu pour le pays des retombées catastrophiques, tous n'attribuent pas cet échec aux mêmes facteurs: pour certains, le caractère excessif de l'endettement était inscrit dans la mesure elle-même, ce qui vouait nécessairement l'Argentine à la crise qui a suivi; pour d'autres, c'est la façon dont la mesure a été mise en œuvre par Domingo Cavallo et le gouvernement, ainsi que des ministres de l'économie qui se sont succédé, qui serait en cause. 
D’ailleurs, Roque Pérez, le personnage principal de La última caravana, ne joue pas les incorruptibles, il n'a pas la rectitude morale du policier Lascano, créé par Eduardo Mallo:

De golpe me imaginé lo que sería como ministro, o presidente, sometido a las presiones de las multinacionales que me mandaban Ferraris de regalo, o cuentas secretas en Suiza, o mujeres fuera de serie. No daba un escarbadientes usado por mi integridad moral (Argemí 2008: 121).

Même constat pour le personnage de Nadie ama a un policía:

Usted puede ser el tipo más derecho del mundo, pero si el clima a su alrededor es de corrupción y los corruptos no van en cana sino que se montan a los mejores autos y a las mejores mujeres, tarde o temprano terminará echando su sistema de valores por el inodoro, con perdón de la metáfora (Orsi 2007: 126).

Il s'agit d'une crise généralisée, à la fois financière et économique, politique et institutionnelle, sociale aussi bien sûr. La lecture qu'en proposent ces deux romans, ne met-elle pas surtout l'accent sur sa dimension de véritable crise des valeurs morales?

\section{Crise et Dictature}

Il est un aspect dont nous n'avons pas encore parlé, et qui pourtant est omniprésent dans ces textes, ce sont les rapports entre la crise et la violence. La pauvreté subie par la population, conséquence d'un système qui organise la concentration des richesses aux mains d'une minorité bien décidée à perpétuer les inégalités, apparaît en soi comme une violence. Un des personnages de Nadie ama a un policía va jusqu'à comparer les morts dues à la malnutrition, fruit de la paupérisation croissante d'une partie de la population, à un "génocide": «El genocidio está ante nuestras narices»(Orsi 2007: 200). Mais la violence s'exprime également de manière plus directe, tout simplement par la répression meurtrière. C'est alors que s'établit un lien, pas seulement par analogie mais bien plus profond, entre la crise de 2001 et la dernière dictature. La crise se présente dans cette perspective comme un véritable prolongement des années noires du «Proceso».

Si cela apparaît comme une évidence du point de vue économique, dans la mesure où les militaires mènent une politique néolibérale qui culmine dans les années quatre-vingt-dix pour s'effondrer brutalement en 2001, cela l'est moins du point de vue politique. La période voit en effet un régime démocratique succéder à un régime dictatorial et toute une génération grandir dans l'aprèsdictature, ce qui paraissait ancrer durablement l'Argentine dans une démocratie qui vient de fêter ses trente ans. La crise de 2001 fit exploser cette vision des choses lorsque le gouvernement démocratique répondit à un mouvement pacifique par une violence digne de méthodes que l'on voulait croire enterrées depuis 1983. 
Le gouvernement de Cristina Kirchner, lors du bilan de la crise, dix ans après celle-ci, ne manqua pas de faire l'amalgame entre le modèle néolibéral et l'usurpation du pouvoir par les dictatures. Son chef de cabinet, Juan Manuel Abal Medina, déclarait à cette occasion:

No fue simplemente el final de un gobierno, sino que fue el final de un modelo que se inició a sangre y fuego el 24 de marzo de 1976, y que terminó eclosionando en esa impotencia de la política, con esa salvaje represión. (Meyer 2011).

Damián Nabot, dans Dos semanas, cinco presidentes. Diciembre de 2001: la historia secreta, lorsqu'il doit relater le moment où les policiers ont tiré sur la foule pacifique, lors du "cacerolazo", s'exclame:

¿Es posible? Dos décadas después del fin de la dictadura, un gobierno democrático reprime a las Madres de la Plaza de Mayo, símbolo internacional de la lucha contra el autoritarismo? (Nabot 2011).

La fuite de Fernando de la Rúa est comparée chez Guillermo Orsi à celle d'Isabel Perón en 1976, ce qui peut se lire comme une autre façon de mettre en évidence un cycle allant de 1976 à 2001 :

Como Isabel Perón en 1976, a fines de 2001 Fernando de la Rúa huía por los techos de un lugar que se había vuelto peligroso. Un rato antes y mientras el presidente escribía su renuncia, la vergüenza nacional había disparado sobre espontáneos manifestantes como en un safari donde los cazadores se ven rodeados por sus presuntas presas (Orsi 2007: 259).

Interrogé précisément sur ce sujet lors d'une entrevue, Ernesto Mallo exprime dans des termes explicites ce que les deux romans disent de manière implicite: "Cuando se va la dictadura la violencia cambia de manos, pero no desaparece. Se requiere mucho tiempo para acabar la maquinaria de la violencia. ${ }^{6}$ ”

La crise de 2001 semble ainsi avoir rappelé aux Argentins que les dictatures n'ont malheureusement pas le monopole de la violence. La violence peut refaire surface même dans le contexte d'une démocratie, soit de manière brutale, par exemple lorsque la police a tiré sur la foule le 20 décembre 2001, soit de manière larvée, mais non moins nocive. Elle peut même apparaître sous la forme d'une réforme économique, le "uno a uno" comme nous l'avons vu. Pour certains économistes, cette mesure reproduit sur le plan symbolique le dernier coup d'état militaire:

On pourrait même risquer une analogie: la convertibilité définirait une violence symbolique, possible réédition sur le mode mineur de la violence du coup deétat de 1976, qui continuerait ainsi à marquer l'Argentine. (Blaum 2004, cité par Boyer et Neffa).

6 http://www.abretelibro.com/foro/viewtopic.php?f=35\&t=56677 (consulté le 11/12/2013). Les romans d'Ernesto Mallo, relatant les aventures de son héros «Lascano », mettent également en évidence, en tant que série, la façon dont la violence a perduré en Argentine malgré l'avènement de la démocratie. Voir à ce propos l'article "Violencia política, violencia policial en la narrativa de Ernesto Mallo» (Caplán R., Fisbach E 2010) 
Guillermo Orsi développe cette même idée sur le mode hyperbolique et dans des termes plus crus, propres à son écriture policière:

La logística del fraude se ha perfeccionado de tal manera en La Argentina que, si hoy tuviéramos una dictadura, tirar presos vivos al mar sería considerado una chapucería (Orsi 2007: 193).

Là où les économistes voient une reproduction symbolique de la violence "sur le mode mineur», le narrateur de Nadie ama a un policía n'hésite pas à utiliser l'un des symboles les plus marquants de la cruauté des militaires pour décrire le système économique implanté en Argentine.

Dans le roman de Raúl Argemí, le personnage principal rencontre l’inventeur du « uno a uno » : il s'agit d'un poivrot qui, avant Cavallo lui-même, avait conçu le principe de la convertibilité. Il avait en effet imaginé une nouvelle valeur, le "pesólar». Le lecteur est clairement invité à faire le rapprochement entre ce personnage ridicule, tourné en dérision, et le véritable instigateur du principe de convertibilité. Or, ce même inventeur du "pesólar» préconise également l'utilisation de la planche à billets, à l'origine de la fameuse "plata dulce» qui, pendant le «Proceso», a donné aux Argentins une éphémère illusion de prospérité économique, avant de déboucher sur l'hyperinflation qui a ruiné les classes moyennes et plongé les plus pauvres dans une misère encore plus grande.

Ces romans se donnent alors à lire comme le développement de ce constat, à la fois paradoxal et désolant, que la démocratie a apporté à l'Argentine davantage d'horreurs que les militaires pendant la dernière dictature. Pino Solanas, dans son film Memoria de un saqueo, exprime la même amertume:

El país había sido devastado por un nuevo tipo de agresión ejecutada en paz y democracia, dejaba más víctimas sociales, más emigrados y muertos, que los del terrorismo de estado y la guerra de Malvinas. (Solanas 2004).

\section{La crise et le grotesque}

Cette vision de la crise, à la fois réaliste et pessimiste, n'empêche pas les romans de manier l'humour. Ainsi, par exemple, la longue liste de coups d'état militaires qu'a connus le pays, et qui semble paradoxalement culminer par un «coup d'état» civil, donne lieu dans Nadie ama a un policía... au commentaire suivant:

Algún día la Argentina debería plantearse seriamente la exportación de la sofisticada logística necesaria para quitarle el poder a los gobiernos sin esperar a que cumplan con sus periodos constitucionales. Ha sido ensayada y desarrollada durante todo el siglo veinte como en pocas naciones no africanas del mundo, y si es cierto que somos los mejores en todo, no tendríamos competencia (Orsi 2007:296).

L'humour, omniprésent, qui prend ici la forme d'une autocritique et reste assez «bon enfant» malgré l'ironie du propos, prend souvent l'aspect du 
grotesque, inscrivant ainsi ces auteurs dans la lignée d'un Osvaldo Soriano ${ }^{7}$. Par exemple, le personnage principal de Guillermo Orsi est aidé dans son enquête par des personnages aux allures farcesques: un médecin obèse («el médico rechoncho») et deux personnages comparés à Don Quichotte et Sancho Panza, que le narrateur appelle le "dúo cervantino» (Orsi 2007: 192). Dans le roman d'Argemí, le vieux fourgon qui transporte les personnages pendant leur épopée carnavalesque est baptisé «Rocinante» (Argemí 2008: 142). Le rôle du bouffon est incarné par un vieux rockeur raté qui s'affuble d'une perruque ridicule. Les scènes désignées dans les romans comme «fantochadas», "farsas» ou «disparates» sont innombrables.

Soulignons que le grotesque ne relève pas seulement d'un héritage littéraire, il sert en fin de compte à caractériser la nature même de la crise et ses acteurs. "La gente en la calle les grita a los dirigentes políticos "que se vayan todos", pero no se irá nadie [...]», s'exaspère le narrateur de Nadie ama a un policía... (Orsi 2008: 268). Le ballet incessant au sommet du pouvoir donne à ses acteurs des allures de fantoches: la crise devient alors l'illustration du "grotesco nacional », pour reprendre l'expression utilisée dans le roman de Guillermo Orsi pour désigner la façon dont fonctionne la démocratie argentine. Ce «grotesco nacional» si bien dépeint dans son tango "Cambalache» par Discépolo dès 1935! (Orsi 2008: 261/309). Ainsi, le rire provoqué par les multiples scènes comiques ne doit pas être compris comme une dépolitisation du propos, qui tendrait à signifier que finalement, «il vaut mieux en rire». Au contraire, c'est la nature même de la crise qui est visée: lui accoler l'adjectif «grotesque» n’en minimise ni la portée, ni les causes. Ainsi, le grotesque désigne moins dans ces romans une esthétique, un choix narratif, que la réalité elle-même.

\section{Conclusion}

La crise de 2001 apparaît comme le moment où culmine tout un processus longuement montré dans ces romans. Sa mise en récit, par le truchement de la fiction, permet aux auteurs de questionner en profondeur les fondements de la démocratie et, in fine, de l'identité argentine.

Les deux romans sont très peu autoréférentiels: ils s'intéressent davantage à la réalité qu'ils décrivent qu'à leur propre processus d'écriture. Chez Argemí, cet aspect métafictionnel est cependant plus évident, dans la mesure où le lecteur peut être assimilé à la fille du personnage principal qui écoute son père lui raconter son histoire. Le narrateur dit à propos de ce narrataire, qui est donc

7 En cela, Argemí et Orsi possèdent de nombreux points en commun avec l'esthétique des romans de leur compatriote Carlos Salem. Comme eux, Salem est édité par des maisons d'édition espagnoles, et il est relativement méconnu dans son propre pays. Tout comme les œuvres de Carlos Salem, les deux romans sont marqués par l'ironie, la dérision et l'autodérision, l'humour noir, mais aussi et surtout par la dégradation carnavalesque, caractéristiques de toute une nouvelle génération d'auteurs de polars. 
à notre avis tout autant intradiégétique que potentiellement extradiégétique: «A la larga, un oyente atento descubriría las notas falsas, y no tardaría en reemplazarlas por las verdaderas» (Argemí 2007: 69). Ainsi, la fiction policière, tout en se revendiquant ici comme mensonge, n'a de cesse de rechercher une vérité cachée. C'est aussi le sens que l'on peut donner à ces propos d'un des personnages de Nadie ama a un policía: «Pero a veces la realidad se construye desde la ficción" (Orsi 2008: 126). Les deux romans ont en effet construit la réalité de la crise depuis la fiction, et c'est bien le travail du lecteur, que de trouver ce qui, dans le "faux», contient finalement le vrai, même si le narrateur du roman de Guillermo Orsi constate: «no hay manera, ni racional ni estética, de explicar el terror» (2008: 157).

\section{Bibliographie}

Argemí, R., 2008, La última caravana, España, Edebé.

Boyer, R., Neffa, J.C., Miotti, L., Quenan, C., 2004, La economía argentina y sus crisis (1976-2001): visiones institucionalistas y regulacionistas, Buenos Aires, Niño y Dávila - Ceil-Piette.

Boyer, R., Neffa J.C., La crise argentine 1976-2001: Lectures institutionnalistes et régulationnistes, hal.archives-ouvertes.fr/docs/00/58/76/96/PDF/wp200746. pdf. [consultée le 12/12/2013].

Bonasso, M., 2002, El palacio y la calle. Crónicas de insurgentes y conspiradores, Buenos Aires, Planeta.

Caplán, R., Fisbach, E., 2010, «Violencia política, violencia policial en la narrativa de Ernesto Mallo », in: Las armas y las letras, La violencia política en la cultura rioplatense desde los años 60 hasta nuestros días, Presses universitaires de Bordeaux, p. 153-168.

Gobello, J., Oliveri M.H., 2002, Diccionario de la crisis, Buenos Aires, Corregidor.

Jozami, A., 2003, Argentina, la destrucción de una nación, Buenos Aires, Mondadori.

Meyer, A, 2011, «Un homenaje a la resistencia», in: Página 12, Argentine. http:// www.pagina12.com.ar/diario/elpais/1-183792-2011-12-20.html. [consulté le $12 / 12 / 2013]$.

Nabot, D., 2011, Dos semanas, cinco presidentes. Diciembre de 2001: la historia secreta, Buenos Aires, Aguilar.

Orsi, G., 2007, Nadie ama a un policía, España, Almuzara.

Piñeiro, Claudia, 2005, Las viudas de los jueves, Madrid, Alfaguara.

Ponce, Néstor, 2008, Azote, México, Terracota.

Solanas, F. E., 2004, Memoria del saqueo (film documentaire), Argentine.

Suriano, J. (dir.), 2005, Dictadura y Democracia (1976-2001), Buenos Aires, Sudamericana. 
Wieser, D., 2012, Crímenes y sus autores intelectuales: Entrevistas a escritores del género policial en América Latina y África lusófona, Göttingen, GOEDOC, Dokumenten- und Publikationsserver der Georg-August-Universität. 